\title{
Biosynthesis of silver nanoparticles synthesized by Aspergillus flavus and their antioxidant, antimicrobial and cytotoxicity properties
}

\author{
GHASSAN M SULAIMAN*, HIBA T HUSSIEN and MAYSOON M N M SALEEM \\ Biotechnology Division, Applied Science Department, University of Technology, Baghdad 10001, Iraq
}

MS received 24 June 2014

\begin{abstract}
In the present study, biosynthesis of silver nanoparticles and its antioxidant, antimicrobial and cytotoxic activities were investigated. Silver nanoparticles were extracellularly synthesized using Aspergillus flavus and the formation of nanoparticles was observed after $72 \mathrm{~h}$ of incubation. The results recorded from colour changes, UV-vis spectrum and X-ray diffraction (XRD) support the biosynthesis and characterization of silver nanoparticles. UVvis spectral analysis showed silver surface plasmon resonance band at $420 \mathrm{~nm}$. X-ray diffraction showed that the particles were crystalline with face-centred cubic structure at $45.05^{\circ}, 65.45^{\circ}$ and $78.65^{\circ}$ and the size of the silver nanoparticles was $33.5 \mathrm{~nm}$. The synthesized silver nanoparticles showed potent antimicrobial activity against various pathogens, including bacteria and fungi. Biosynthesized silver nanoparticles exhibited strong antioxidant activity as well as cytotoxicity against HL-60 cells in a dose-response relationship. The powerful bioactivity demonstrated by the synthesized silver nanoparticles leads towards the biomedical use as antioxidant, antibacterial and cytotoxic agents.
\end{abstract}

Keywords. Aspergillus flavus; silver nanoparticles; antimicrobial; antioxidant; cytotoxicity.

\section{Introduction}

Nanoparticles with controlled size and composition are of fundamental and technological interest as they provide solutions to technological and environmental challenges in the areas of solar energy conversion, catalysis, medicine and water treatment. Thus, production and application of nanomaterials from 1 to 100 nanometres (nm) is an emerging field of research. ${ }^{1-3}$ In the synthesis of nanoparticles, the various conventional processes like a number of chemical and physical methods including chemical reduction in aqueous or non-aqueous solution, ${ }^{4}$ microemulsion, ${ }^{5}$ sono-chemical ${ }^{6}$ and microwave-assisted ${ }^{7}$ methods have been applied.

Since the chemicals like organic solvents, hydrazine, sodium borohydride and N,N-dimethyl formamide used in the synthesis of metal nanoparticles are found to be highly reactive and biologically hazardous, the chemically synthesized metal nanoparticles could not be accomplished with the biomedical field. On the other hand, the microbiologically synthesized metal nanoparticles are found to be ecofriendly, reliable, biocompatible and economic. It is reported from the ancient time that silver among the various metals has been considered as an effective antimicrobial agent, food preservative agent and water purifying agent. ${ }^{8,9}$ Accordingly bacteria, ${ }^{10}$ actinomycetes, ${ }^{11}$ plants ${ }^{12,13}$ and fungi ${ }^{9,14}$ have been used for the study of biosynthesis of silver nanoparticles AgNPs, which are of higher production yields and with low expenses.

\footnotetext{
*Author for correspondence (gmsbiotech@ hotmail.com)
}

The use of fungi in the synthesis of nanoparticles is a relatively recent addition to the list of microorganisms possessing nanoparticle biosynthesis. Fungi are ideal candidates in the synthesis of metal nanoparticles, because of their ability to secrete large amount of enzymes. ${ }^{15}$ An interesting example of the biosynthesis using fungi was that the cellassociated biosynthesis of silver using Fusarium oxysporum was demonstrated by Ahmad et al, ${ }^{16}$ and the particles were overall quasi-spherical with size range between 5 and 15 $\mathrm{nm}$. There also have been several reports on the biosynthesis of AgNPs using fungi, including Trichoderma reesei, ${ }^{17}$ Penicillium fellutanum ${ }^{15}$ and Aspergillus sp. ${ }^{18,19}$

In spite of the cost-effective process, report on the use of the fungal strains like Aspergillus sp. for the biosynthesis of AgNPs extracellularly are found to be very scanty. In this regard, we investigate the use a fungi Aspergillus flavus in synthesis of extracellular of AgNPs. Further these biologically synthesized nanoparticles were found highly toxic against different pathogenic microorganisms tested. Antioxidant and toxicity against human acute promyelocytic leukaemia (HL-60) cell line were also evaluated.

\section{Materials and methods}

\subsection{Materials}

The chemical silver nitrate $\left(\mathrm{AgNO}_{3}\right)$, brain heart infusion agar (BHIA) and Sabouraud dextrose agar (SDA, Oxoid) were purchased from Merck, Germany. Penicillin and streptomycin were purchased from Bio Source International, 
Belgium. Tissue culture plastic wares were obtained from BD Bioscience (USA). All organic solvents used were of HPLC grade. RPMI 1640, foetal bovine serum (FBS) and MTT (3-(4,5-dimethyl-thiazol-2-yl)-2,S-diphenyltetrazolium bromide) were purchased from Sigma Chemical Co. (St. Louis, MO, USA). 2,2-Diphenyl-1-picrylhydrazyl (DPPH) (Sigma-Aldrich) was used for the spectrophotometrical determination of free radical scavenging activity.

\subsection{Organism}

The identified strain, A. flavus was used for the study of biosynthesis of AgNPs. Proteus volgaris, Escherichia coli, Pseudomonas aeruginosa (Gram negative) and Staphylococcus aureus (Gram positive), Aspergillus niger, Penicillium chrysogenum, Alternaria alternata and Fusarium culmorum were used to evaluate the antimicrobial activity of prepared AgNPs. These microbes were kindly supplied by the Biotechnology Division, Department of Applied Science, University of Technology, Baghdad, Iraq.

\subsection{Synthesis of AgNPs}

For synthesis of AgNPs from the biomass, A. flavus which cultured on Saboroud dextrose agar (SDA) plate, then the colonies were grown in $250 \mathrm{ml}$ Erlenmeyer flask containing $200 \mathrm{ml}$ nutrient broth medium which composed of peptic digest of animal tissue $\left(5 \mathrm{~g} \mathrm{l}^{-1}\right)$, beef extract $\left(1.50 \mathrm{~g} \mathrm{l}^{-1}\right)$, yeast extract $\left(1.50 \mathrm{~g} \mathrm{l}^{-1}\right)$ and sodium chloride $\left(5.00 \mathrm{~g}^{-1}\right)$. This culture was incubated on orbital shaker with $150 \mathrm{rpm}$ agitation at $28^{\circ} \mathrm{C}$ for $96 \mathrm{~h}$. After $96 \mathrm{~h}$ incubation, fungal biomass was separated from nutrient broth by centrifuging at $3500 \mathrm{rpm}$ at $10^{\circ} \mathrm{C}$ for $20 \mathrm{~min}$ and washed with distilled water to remove any medium components. Fresh and clean biomass was exposed in $100 \mathrm{ml}$ of $2 \mathrm{mM}$ aqueous $\mathrm{AgNO}_{3}$ solution with pH 6.5 in $250 \mathrm{ml}$ Erlenmeyer flask. The whole mixture was put into a shaker at $28^{\circ} \mathrm{C}$ for $72 \mathrm{~h}$ in dark. The sample was characterized and examined for antioxidant, antimicrobial and cytotoxic activities.

\subsection{Characterization of AgNPs}

UV-vis spectral analysis was carried out by using UV-vis spectrophotometer $\left(\mathrm{PG}-\mathrm{T} 80^{+} \mathrm{UV} / \mathrm{vis}\right.$ spectrophotometer, England) from 300 to $600 \mathrm{~nm}$ at a resolution of $1 \mathrm{~nm}$. $\mathrm{X}$-ray diffraction (XRD) measurements of the silver nanoparticles solution drop-coated on glass were done on a Shimadzu XRD-6000 model with $40 \mathrm{kV}, 30 \mathrm{~mA}$ with $\mathrm{Cu} \mathrm{K} \alpha$ radiation at $2 \theta$ angle. $\mathrm{X}$-ray powder diffraction is a rapid analytical technique primarily used for phase identification of a crystalline material and can provide information on unit cell dimensions. The crystallite domain size was calculated from the width of the XRD peaks, assuming that they are free from non-uniform strains, using the Scherrer formula

$$
D=0.94 \lambda / \beta \cos \theta .
$$

\subsection{Measurements of antioxidant activity}

Antioxidant activity of AgNPs was measured by the DPPH method. One millilitre of $0.1 \mathrm{mM}$ DPPH (in ethanol) was added to different concentrations (100, 200 and $400 \mu \mathrm{g} \mathrm{ml}^{-1}$ ) of AgNPs synthesized by A. flavus. The reaction mixture was shaken and incubated in the dark for $30 \mathrm{~min}$. The absorbance at $517 \mathrm{~nm}$ was measured against a blank (ethanol). The lower absorbance of the reaction mixture indicated a higher percentage of scavenging activity. DPPH scavenging activity is calculated using the following equation:

$$
\text { DPPH scavenging activity }(\%)=\left(\frac{A_{\mathrm{c}}-A_{\mathrm{s}}}{A_{\mathrm{c}}}\right) \times 100
$$

where $A_{\mathrm{c}}$ and $A_{\mathrm{s}}$ are the intensity of peak at $517 \mathrm{~nm}$ for control (DPPH) and supernatant DPPH solvent, respectively.

\subsection{Evaluation of antimicrobial activity}

The AgNPs synthesized using A. flavus was tested for antimicrobial activity by the agar disc diffusion method for pathogenic bacteria and well diffusion method for fungi. The pure cultures of bacteria were sub-cultured on brain BHIA. Each strain was swabbed uniformly onto the individual plates, using sterile cotton swabs. For bacteria, discs of $8 \mathrm{~mm}$ diameter containing the prepared nanoparticle solution were put on mannitol salt agar and nutrient agar medium and incubated at $37^{\circ} \mathrm{C}$ for $24 \mathrm{~h}$. For fungi, wells with a diameter of $8 \mathrm{~mm}$ were made on SDA plates by using the gel puncture method. A micropipette was used to pour $50 \mu \mathrm{l}$ of prepared nanoparticle solution onto each well on all plates and incubated at $30^{\circ} \mathrm{C}$ for 3-7 days. For each experiment, the diameter of zone inhibition was measured in millimetre, and was recorded as mean $\pm \mathrm{SD}$ of the triplicate experiment.

\subsection{Statistical analysis}

The grouped data were statistically evaluated using ANOVA with SPSS/14 software. Values are presented as the mean \pm $\mathrm{SD}$ of the three replicates of each experiment.

\section{Results and discussion}

\subsection{Synthesis and characterization of AgNPs using A. flavus}

In this study, AgNPs were synthesized using a reduction of aqueous $\mathrm{Ag}^{+}$with the culture supernatants of $A$. flavus at room temperature. It was generally recognized that AgNPs produced brown solution in water. These characteristic colour variations are due to the excitation of the surface plasmon resonance in the metal nanoparticles. ${ }^{20}$ After 


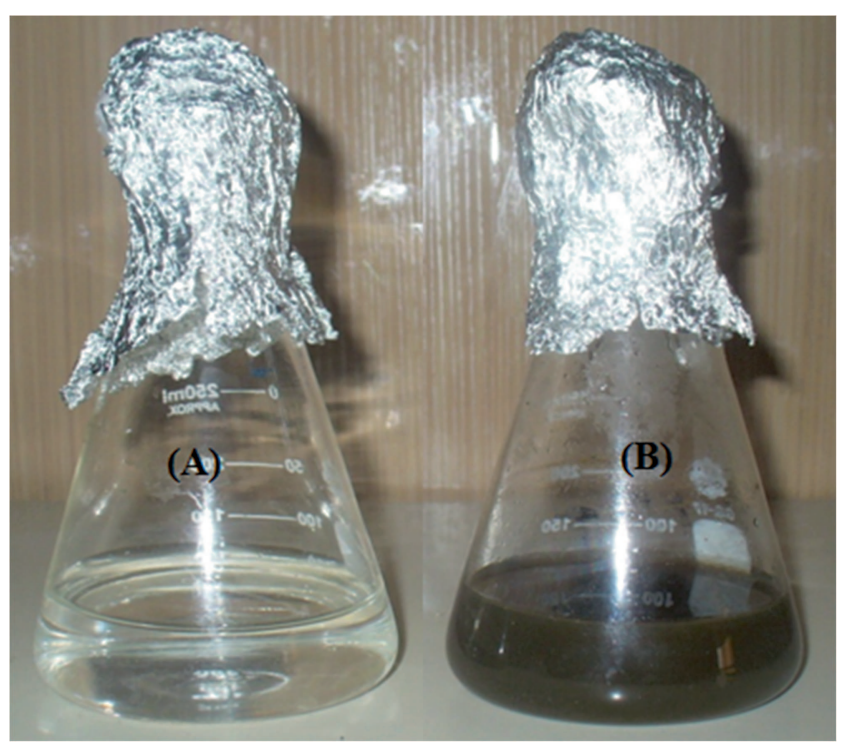

Figure 1. The change of colour from colourless (A) to brownish (B) of live cell filtrate of Aspergillus flavus after the addition of $\mathrm{AgNO}_{3}$ solution $(2 \mathrm{mM})$.

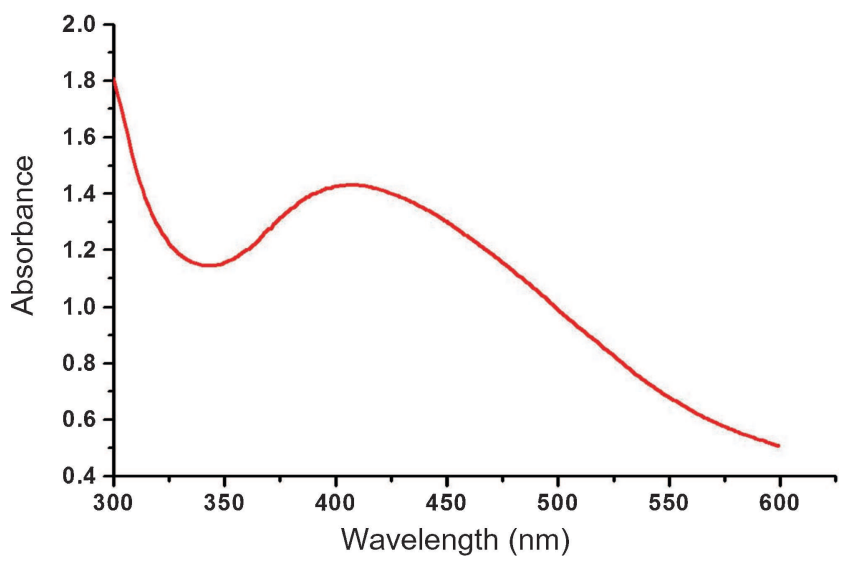

Figure 2. UV/vis absorption spectra of reduction of silver ions to silver nanoparticles after $72 \mathrm{~h}$ of reaction. the addition of $\mathrm{AgNO}_{3}$ solution, the crude cell filtrate of $A$. flavus changed from light yellow to brown in a $72 \mathrm{~h}$, while no colour change was observed in the culture supernatant without $\mathrm{AgNO}_{3}$ (figure 1). Thus, colour change of the solution clearly indicated the formation of AgNPs. The colour intensity of the cell filtrate with $\mathrm{AgNO}_{3}$ was sustained even after $24 \mathrm{~h}$ of preparation, which indicated that the particles were well dispersed in the solution, and there was no obvious aggregation.

Formation of AgNPs was confirmed using UV-vis spectral analysis and showed silver surface plasmon resonance band at $420 \mathrm{~nm}$ (figure 2). The frequency and width of the surface plasmon absorption depend on the size and shape of the metal nanoparticles as well as on the dielectric constant of the metal itself and the surrounding medium. ${ }^{21}$ It is generally recognized that $\mathrm{UV}$-vis spectroscopy could be used to examine size- and shape-controlled nanoparticles in aqueous suspensions. $^{22}$

The XRD pattern of the biosynthesized silver nanostructure synthesized by the A. flavus was further demonstrated and confirmed by the characteristic peaks observed in the XRD image (figure 3). The Braggs reflections were observed in the XRD pattern at $2 \theta$ were $45.05^{\circ}, 65.45^{\circ}$ and $78.65^{\circ}$ in the whole spectrum of value ranging from 10 to 90 and indicated that the structure of AgNPs is face-centred cubic (fcc). These are corresponding to [200], [220] and [311] planes for silver, respectively. Some intense diffraction peaks at $2 \theta$ angles of $38.45^{\circ}, 38.77^{\circ}$ might be related to $\mathrm{AgCl}$ which was owing to the chloride ions involved during the preparation of the cell filtrate. The lattice constant calculated from this pattern was $a=4.086 \AA$ and the data obtained was matched with the database of Joint Committee on Powder Diffraction Standards (JCPDS) file no. 04-0783. The average grain size of the AgNPs formed in the bioreduction process was determined using Scherrer's formula and was estimated as $33.5 \mathrm{~nm}$. The XRD results clearly show that the AgNPs formed by the reduction of $\mathrm{Ag}^{+}$ions by the A. flavus might are crystalline in nature.

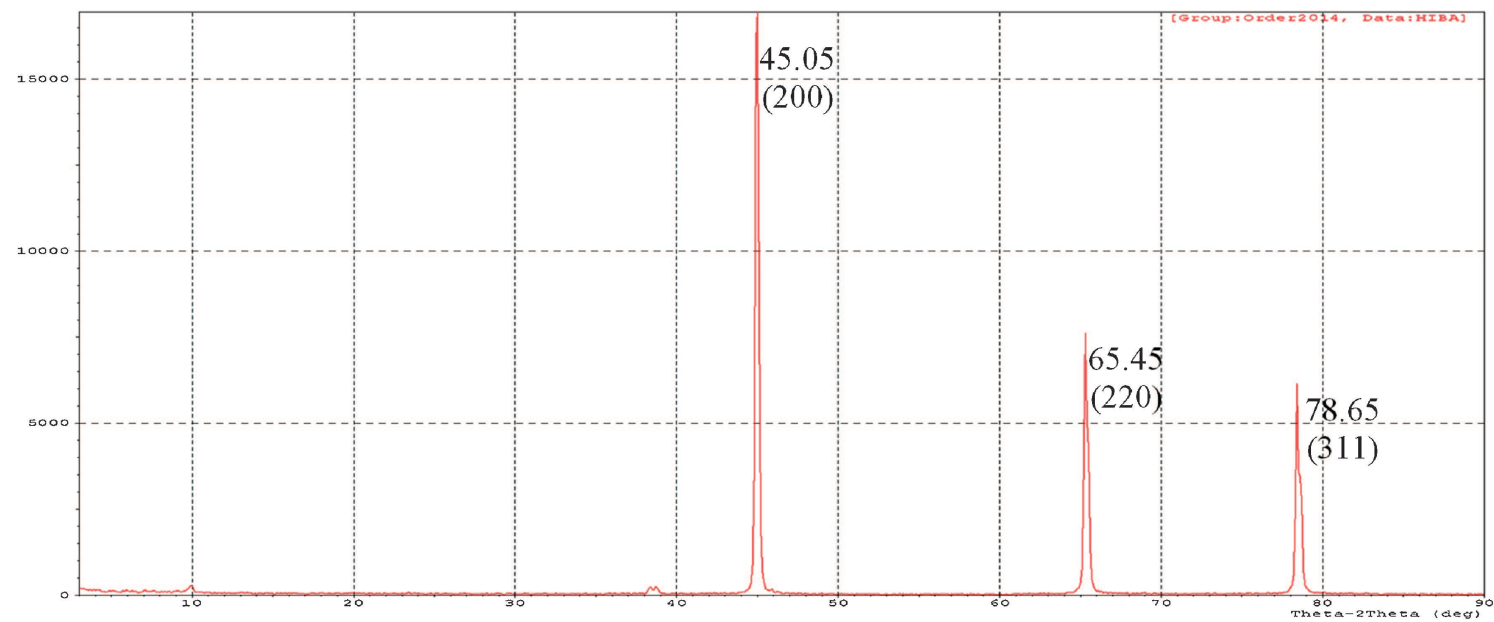

Figure 3. XRD pattern of silver nanoparticles formed after reaction of Aspergillus flavus. 


\subsection{Free radical scavenging activity of AgNPs}

In this study we investigated the free radical scavenging activities of AgNPs on the basis of their capability to bleach the stable radical DPPH. The DPPH assay provided information on the reactivity of the test compounds with a stable free radical. DPPH gives a strong absorption band at $517 \mathrm{~nm}$ in visible spectroscopy (deep violet colour) because of its odd electron. Given that this electron becomes paired off in the presence of a free radical scavenger, the absorption vanishes, and the resulting decolourization is stoichiometric with respect to the number of electrons taken up. ${ }^{23,24}$ As shown in figure 4, the free radical scavenging effect of AgNPs in DPPH was determined for 100, 200 and $400 \mu \mathrm{g} \mathrm{ml}^{-1}$ and show concentration-dependent activities. The concentration of $400 \mu \mathrm{g} \mathrm{ml}^{-1}$ was significantly better than the other two concentrations. Superoxide anions are free radicals generated by the transfer of one electron and play an important role in the formation of other reactive oxygen species such as hydrogen peroxide, hydroxyl radical or singlet oxygen in living systems. ${ }^{25}$ Due to their scavenging power, antioxidants are useful for the management of diseases such as neurodegenerative diseases, cancer and AIDS. Antioxidants can also react with nitric oxide to form peroxynitrite, which can generate toxic radicals such as the hydroxyl radical. ${ }^{26}$

\subsection{Antimicrobial activity of AgNPs}

In this study, the application of AgNPs as an antimicrobial agent was investigated and exhibited better antimicrobial activity against all pathogens. Compared with the control, the diameters of inhibition zones increased for all

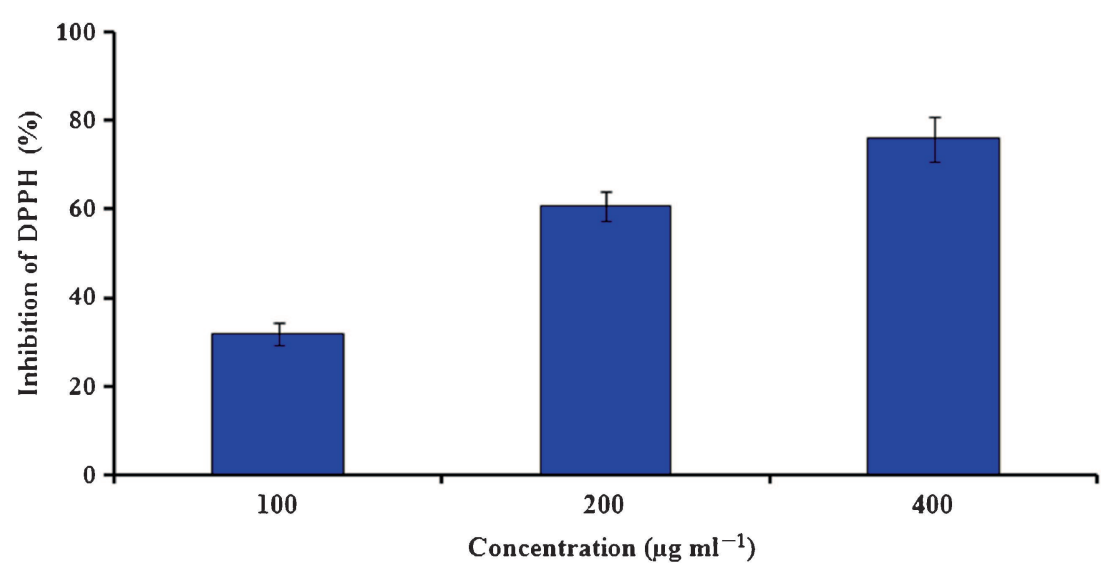

Figure 4. DPPH free radical scavenging activity of silver nanoparticles formed after reaction of Aspergillus flavus. Results are expressed as percentage decrement of absorbance at $517 \mathrm{~nm}$ with respect to control. Each value represents the mean $\pm \mathrm{SD}$ of three experiments.

Table 1. Zone of inhibition of silver nanoparticles synthesized by Aspergillus flavas against pathogenic bacteria.

\begin{tabular}{lrrrr}
\hline & \multicolumn{4}{c}{ Zone of inhibition (mm) } \\
\cline { 2 - 5 } Pathogen & \multicolumn{1}{c}{$\mathrm{AgNO}_{3}$} & $5 \mu \mathrm{g} \mathrm{ml}^{-1}$ & $10 \mu \mathrm{g} \mathrm{ml}^{-1}$ & $20 \mu \mathrm{g} \mathrm{ml}^{-1}$ \\
\hline Proteus volgaris & $8.0 \pm 0.0$ & $16.3 \pm 0.6$ & $20.6 \pm 0.6$ & $26.6 \pm 0.6$ \\
Escherichia coli & $9.0 \pm 0.0$ & $11.5 \pm 0.5$ & $16.3 \pm 0.6$ & $22.3 \pm 0.6$ \\
Pseudomonas aeruginosa & $9.5 \pm 0.5$ & $13.6 \pm 0.6$ & $18.5 \pm 0.5$ & $23.3 \pm 0.6$ \\
Staphylococcus aureus & $10.5 \pm 0.5$ & $25.6 \pm 0.6$ & $26.0 \pm 0.0$ & $28.5 \pm 0.5$ \\
\hline
\end{tabular}

Table 2. Zone of inhibition of silver nanoparticles synthesized by Aspergillus flavus against pathogenic fungi.

\begin{tabular}{lllll}
\hline & \multicolumn{4}{c}{ Zone of inhibition (mm) } \\
\cline { 2 - 5 } Pathogen & $\mathrm{AgNO}_{3}$ & $5 \mu \mathrm{g} \mathrm{ml}^{-1}$ & $10 \mu \mathrm{g} \mathrm{ml}^{-1}$ & $20 \mu \mathrm{g} \mathrm{ml}^{-1}$ \\
\hline Aspergillus niger & $8.0 \pm 0.0$ & $20.1 \pm 0.1$ & $22.3 \pm 0.6$ & $30.2 \pm 0.1$ \\
Penicillium chrysogenum & $8.0 \pm 0.0$ & $18.2 \pm 0.1$ & $22.0 \pm 0.5$ & $28.5 \pm 0.5$ \\
Alternaria alternata & $9.0 \pm 0.0$ & $20.3 \pm 0.6$ & $22.6 \pm 0.6$ & $30.8 \pm 0.4$ \\
Fusarium culmorum & $8.0 \pm 0.0$ & $20.1 \pm 0.1$ & $21.8 \pm 0.3$ & $24.0 \pm 0.5$ \\
\hline
\end{tabular}


the test pathogens. However, the antimicrobial effect was dose-dependent response (tables 1 and 2 and figure 5). The AgNPs produced could inhibit three different typical pathogenic bacteria, including Staphylococcus aureus, Pseudomonas aeruginosa and Escherichia coli, as previously described. ${ }^{19,27}$ Thus, AgNPs could be considered as excellent broad-spectrum antibacterial agents.

Silver has been used for many years for antimicrobial applications in the medical field. In recent times, in the field of nanotechnology, applications of AgNPs as antimicrobial agents have been expanding. The exact mechanism by which silver ions and AgNPs exert their antibacterial effect remains to be identified. A literature survey shows that smaller AgNPs having a large surface area available for interaction would have a stronger antibacterial effect than larger AgNPs. It is also possible that AgNPs not only interact with the membrane surface, but may also penetrate inside bacteria. More importantly, the AgNPs produced by A. flavus exhibited potent antifungal activity against the tested pathogenic fungi (table 2). Additionally, AgNPs showed good inhibition activity towards two kinds of filamentous fungus, which were naturally resistant to the common antifungal agent fluconazole. ${ }^{28}$ Since the biosynthesized AgNPs showed

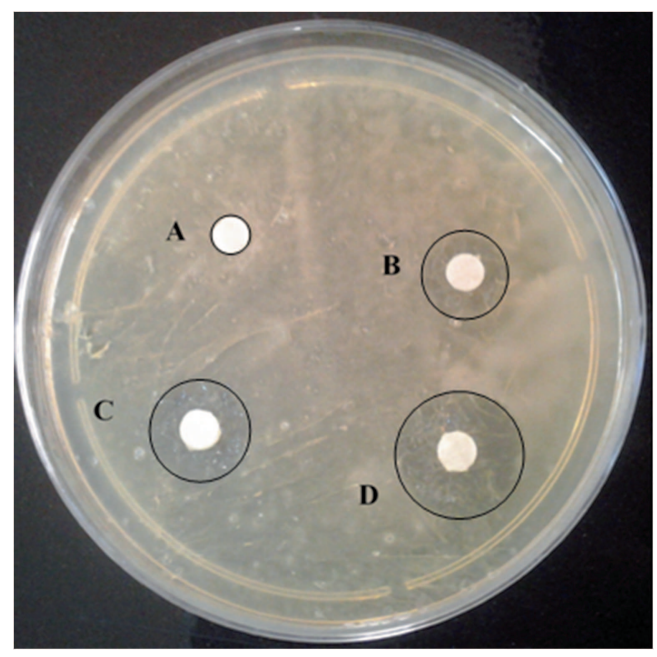

Figure 5. Antibacterial activity assay against Proteus volgaris by the disk diffusion method: (A) silver nitrate, (B) synthesized silver nanoparticles at $5 \mu \mathrm{g} \mathrm{ml}^{-1}$, (C) synthesized silver nanoparticles at $10 \mu \mathrm{g} \mathrm{ml}^{-1}$ and (D) synthesized silver nanoparticles at $20 \mu \mathrm{g} \mathrm{ml}^{-1}$. considerable antifungal activity, they could be potential to be widely used in clinical applications.

\subsection{Antiproliferative activity of AgNPs in HL-60}

The antiproliferative activity of AgNPs was evaluated in human promyeloid leukaemia cells (HL-60). As shown in table 3, treatment with AgNPs at concentrations of 5 and $10 \mu \mathrm{g} \mathrm{ml}^{-1}$ showed a dose- and time-dependent decrease in cell viability. The viability of tumour cells after incubation with AgNPs were greatly affected ( $88 \pm 4.44 \%$ cell death) compared with untreated cells (2\% cell death) after $24 \mathrm{~h}$ of incubation (table 3 ). The mortality data obtained in these results allow us to predict their potential not only because of the cytotoxic effect, but also in terms of the potential for tumour reduction. The toxicity of AgNPs appeared much higher than that of $\mathrm{AgNO}_{3}(21 \pm 2.60 \%)$ at the same period of incubation (table 3 ). The cytotoxic effects of silver are the result of active physicochemical interaction of silver atoms with the functional groups of intracellular proteins, as well as with the nitrogen bases and phosphate groups in DNA. ${ }^{29,30}$ This may be due to their inhibitory activities in several signaling cascades responsible for the development and pathogenesis of the disease which are as yet not understood. AgNPs have been shown to induce the apoptotic pathway in vitro through free oxygen radical generation, which showed antitumour, antiproliferative and antiangiogenic effects in vitro. ${ }^{31,32}$

AgNPs are gaining much interest among the emerging nanoproducts in the field of nanomedicine due to their unique properties and obvious therapeutic potential in treating a variety of diseases, including retinal neovascularization. ${ }^{31}$ AshaRani et $a l^{32}$ reported that AgNPs inhibit proliferation of human glioblastoma cells. Franco-Molina et $a l^{33}$ evaluated the effects of colloidal silver on MCF-7 human breast cancer cells. Sanpui et $a l^{34}$ demonstrated that AgNPs not only disrupted normal cellular function, but also affected membrane integrity, inducing various apoptotic signalling genes of mammalian cells, leading to programmed cell death.

\section{Conclusion}

The mortality data obtained in present study allow us to predict their potential not only because of the cytotoxic

Table 3. Percent viability measured on HL-60 cells for 6,12 and 24 h, by MTT assay.

\begin{tabular}{|c|c|c|c|c|c|c|}
\hline \multirow[b]{3}{*}{ AgNPs } & \multicolumn{4}{|c|}{ Time of incubation $(\mathrm{h})^{\mathrm{a}}$} & & \\
\hline & \multicolumn{2}{|c|}{6} & \multicolumn{2}{|c|}{12} & \multicolumn{2}{|c|}{24} \\
\hline & Dead $(\%)$ & Viable $(\%)$ & Dead $(\%)$ & Viable $(\%)$ & Dead $(\%)$ & Viable $(\%)$ \\
\hline HL-60 + RPMI & $3 \pm 0.12$ & 97 & $2 \pm 0.24$ & 98 & $2 \pm 0.16$ & 98 \\
\hline $\mathrm{AgNO}_{3}$ & $8 \pm 1.30$ & 92 & $15 \pm 1.44$ & 85 & $21 \pm 2.60$ & 79 \\
\hline $5 \mu \mathrm{g}$ AgNPs & $30 \pm 2.40$ & 70 & $48 \pm 2.82$ & 52 & $58 \pm 2.30$ & 42 \\
\hline $10 \mu \mathrm{g}$ AgNPs & $64 \pm 4.18$ & 36 & $70 \pm 4.21$ & 30 & $88 \pm 4.44$ & 12 \\
\hline
\end{tabular}

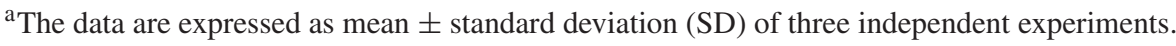


effect on HL-60 cells, but also in terms of the potential for tumour reduction. Therefore, further studies are needed to fully characterize the toxicity and the mechanisms of cell death involved with the antioxidant and cytotoxic activities of these particles.

\section{References}

1. Sun Y G and Xia Y N 2002 Science 2982176

2. Groneberg D A, Giersig M, Welte T and Pison U 2006 Curr. Drug Targets 7643

3. White R J, Budarin V L, Moir J W B and Clark J H 2011 Int. J. Mol. Sci. 125782

4. Petit C, Lixon P and Pileni M P 1993 J. Phys. Chem. 9712974

5. Solanki J N and Murthy Z V P 2010 Colloids Surf. B 35931

6. Korotchenkov O A, Cantarero A, Shpak A P, Kunitskii Y A, Senkevich A I, Borovoy M O and Nadtochii A B 2005 Nanotechnology 162033

7. Nadagouda M N, Speth T F and Varma R S 2011 Acc. Chem. Res. 44469

8. Silver S 2003 FEMS Microbiol. Rev. 27341

9. Roy S, Mukherjee T, Chakraborty S and Kumardas T 2013 Dig. J. Nanomater. Bios. 8197

10. Shahverdi A R, Minaeian S, Shahverdi H R, Jamalifar H and Nohi A A 2007 Proc. Biochem. 42919

11. Ahmad A, Senapati S, Khan M I, Kumar R, Ramani R and Sastry M 2003 Nanotechnology 14824

12. Binupriya A R, Sathishkumar M and Yun S I 2010 Ind. Eng. Chem. Res. 49852

13. Sulaiman G M, Mohammad A AW, Abdul-Wahed H E and Ismail M M 2013 Dig. J. Nanomater. Bios. 8273

14. Ingle A, Gade A, Pierrat S, Sonnichsen C and Rai M 2008 Curr. Nanosci. 4141

15. Kathiresan K, Manivannan S, Nabeel M A and Dhivya B 2009 Colloids Surf. B 71133

16. Ahmad A, Mukherjee P, Senapati S, Mandal D, Khan M I, Kumar R and Sastry R 2003 Colloids Surf. B 28313
17. Vahabi K, Mansoori G A and Karimi S 2011 Insci. J. 165

18. Bhainsa K C and D'Souza S F 2006 Colloids Surf. B 47 160

19. Verma V C, Kharwar R N and Gange A C 2010 Nanomedicine 533

20. Kouvaris P, Delimitis A, Zaspalis V, Papadopoulos D, Tsipas S A and Michailidis N 2012 Mater. Lett. 7618

21. Udayasoorian C, Kumar R V and Jayabalakrishnan M 2011 Dig. J. Nanomater. Bios. 6279

22. Wiley B J, Im S H, Li Z Y, Mc Lellan J, Siekkinen A and Xia Y 2006 J. Phys. Chem. B 11015666

23. Rice-Evans C A, Miller N J and Paganga G 1996 Free Radic. Biol. Med. 20933

24. Sulaiman G M, Al-Amiery A A and Bagnati R 2014 Int. J. Nutr. Food Sci. 65101

25. Stief T W 2003 Med. Hypoth. 60567

26. Halliwell B 1997 Nutr. Rev. 5544

27. Khan S S, Mukherjee A and Chandrasekaran N 2011 Colloids Surf. B 201187129

28. Espinel-Ingroff A, Warnock D W, Vazquez J A and Arthington-Skaggs B A 2000 Med. Mycol. 38293

29. Moaddab S, Ahari H, Shahbazzadeh D, Motallebi A A, Anvar A A, Rahman-Nya J and Shokrgozar M R 2011 Int. Nano Lett. 111

30. Satyavani K, Gurudeeban S, Ramanathan $\mathrm{T}$ and Balasubramanian T 2011 J. Nanobiotechnol. 92

31. Gurunathana S, Leeb K J, Kalishwaralala K, Sheikpranbabua S, Vaidyanathana R and Eom S H 2009 Biomaterials 30 6341

32. AshaRani P V, Low Kah Mun G, Hande M P and Valiyaveettil S 2009 ACS Nano 3279

33. Franco-Molina M A, Mendoza-Gamboa E, Sierra-Rivera C A, Gómez-Flores R A, Zapata-Benavides P, Castillo-Tello P, Alcocer-González J M, Miranda-Hernández D F, TamezGuerra R S and Rodríguez-Padilla C 2010 J. Exp. Clin. Cancer Res. 29148

34. Sanpui P, Chattopadhyay A and Ghosh S S 2011 ACS Appl. Mater. Interface 3218 